\title{
A Modified Particle Swarm Optimization Technique for Economic Load Dispatch with Valve-Point Effect
}

\author{
Hardiansyah \\ Department of Electrical Engineering, University of Tanjungpura, Jl. A. Yani Potianak (78124), West Kalimantan, \\ Indonesia \\ E-mail: hardi_eka@yahoo.com
}

\begin{abstract}
This paper presents a new approach for solution of the economic load dispatch (ELD) problem with valve-point effect using a modified particle swarm optimization (MPSO) technique. The practical ELD problems have non-smooth cost function with equality and inequality constraints, which make the problem of finding the global optimum difficult when using any mathematical approaches. In this paper, a modified particle swarm optimization (MPSO) mechanis $m$ is proposed to deal with the equality and inequality constraints in the ELD problems through the application of Gaussian and Cauchy probability distributions. The MPSO approach introduces new diversification and intensification strategy into the particles thus preventing PSO algorithm from premature convergence. To demonstrate the effectiveness of the proposed approach, the numerical studies have been performed for three different test systems, i.e. six, thirteen and forty generating unit systems, respectively. The results shows that performance of the proposed approach reveal the efficiently and robustness when compared results of other optimization algorithms reported in literature.
\end{abstract}

Index Terms - Particle Swarm Optimization, Economic Load Dispatch, Non-Smooth Cost Functions, Valve-Point Effect

\section{Introduction}

Most of power system optimization problems including economic load dispatch (ELD) which have complex and nonlinear characteristics with heavy equality and inequality constraints. The objective of the ELD of electric power generation is to schedule the committed generating unit outputs so as to meet the required load demand at minimum operating cost while satisfying all unit and system equality and inequality constraints. Several classical optimization techniques such as lambda iteration method, gradient method, Newton's method, linear programming, Interior point method and dynamic programming have been used to solve the basic economic dispatch problem [1]. These mathematical methods require incremental or marginal fuel cost curves which should be monotonically increasing to find global optimal solution. In reality, however, the input-output characteristics of generating units are non-convex due to valve-point loadings and multi-fuel effects, etc. Also there are various practical limitations in operation and control such as ramp rate limits and prohibited operating zones, etc. Therefore, the practical ELD problem is represented as a nonconvex optimization problem with equality and inequality constraints, which cannot be solved by the traditional mathematical methods. Dynamic programming method [2] can solve such types of problems, but it suffers from so-called the curse of dimensionality. Over the past few decades, as an alternative to the conventional mathe matical approaches, many salient methods have been developed for ELD problem such as genetic algorithm [3, 4], improved tabu search [5], simulated annealing [6], neural network [7, 8], evolutionary programming [9]-[11], and particle swarm optimization [14]-[17].

Recently, Kennedy and Eberhart suggested a particle swarm optimization (PSO) based on the analogy of swarm of bird and school of fish. In PSO, each individual makes its decision based on its own experience together with other individual's experiences [12]. The individual particles are drawn stochastically towards the position of present velocity of each individual, their own previous best performance, and the best previous performance of their neighbors. It was developed through simulation of a simplified social system, and has been found to be robust in solving continuous non-linear optimization problems [13]. The main advantages of the PSO algorithm are summarized as: simple concept, easy implementation, and computational efficiency when compared with mathe matical algorithm and other heuristic optimization techniques.

In this paper, a novel approach is proposed to solve the non-smooth ELD problem with valve-point effect using a MPSO technique. The application of Gaussian and Cauchy probability distributions into the PSO is a useful strategy to ensure convergence of the particle swarm algorithm. Feasibility of the proposed MPSO 
method has been demonstrated on three different test systems, i.e. six, thirteen, and forty generating unit systems. The results obtained with the proposed method were analyzed and compared other optimization results reported in literature.

\section{Economic Load Dispatch Formulation}

\subsection{Economic Load Dispatch (ELD) Problem}

The objective of an ELD problem is to find the optimal combination of power generations that minimizes the total generation cost while satisfying equality and inequality constraints. The fuel cost curve for any unit is assumed to be approximated by segments of quadratic functions of the active power output of the generator. For a given power system network, the problem may be described as optimization (minimization) of total fuel cost as defined by (1) under a set of operating constraints.

$$
F_{T}=\sum_{i=1}^{n} F\left(P_{i}\right)=\sum_{i=1}^{n}\left(a_{i} P_{i}^{2}+b_{i} P_{i}+c_{i}\right)
$$

Where $F_{T}$ is total fuel cost of generation in the system $(\$ / \mathrm{hr}), a_{i}, b_{i}$, and $c_{i}$ are the cost coefficient of the $i$ th generator, $P_{i}$ is the power generated by the $i$ th unit and $n$ is the number of generators.

The total generation cost is minimized subjected to the following constraints:

Power balance constraint,

$$
P_{i, \min } \leq P_{i} \leq P_{i, \max } \text { for } i=1,2, \cdots, n
$$

Generation capacity constraint,

$$
P_{D}=\sum_{i=1}^{n} P_{i}-P_{\text {Loss }}
$$

where $P_{i, \min }$ and $P_{i, \text { max }}$ are the minimum and maximum power output of the $i$ th unit, respectively. $P_{D}$ is the total load demand and $P_{\text {Loss }}$ is total transmission losses. The transmission losses $P_{\text {Loss }}$ can be calculated by using $\mathbf{B}$ matrix technique and is defined by (4) as,

$$
P_{\text {Loss }}=\sum_{i=1}^{n} \sum_{j=1}^{n} P_{i} B_{i j} P_{j}+\sum_{i=1}^{n} B_{0 i} P_{i}+B_{00}
$$

where $B_{i j}$ is coefficient of trans mission losses.

\subsection{ELD Problem Considering Valve-Point Effects}

For more rational and precise modeling of fuel cost function, the above expression of cost function is to be modified suitably. The generating units with multivalve steam turbines exhibit a greater variation in the fuel-cost functions [15]. The valve opening process of multi-valve steam turbines produces a ripple-like effect in the heat rate curve of the generators. These "valvepoint effects" are illustrated in Fig. 1.

The significance of this effect is that the actual cost curve function of a large steam plant is not continuous but more important it is non-linear. The valve-point effects are taken into consideration in the ELD problem by superimposing the basic quadratic fuel-cost characteristics with the rectified sinusoid component as follows:

$$
\begin{aligned}
F_{T} & =\sum_{i=1}^{n} F\left(P_{i}\right) \\
& =\sum_{i=1}^{n}\left(\begin{array}{l}
a_{i} P_{i}^{2}+b_{i} P_{i}+c_{i}+ \\
\left|e_{i} \times \sin \left(f_{i} \times\left(P_{i, \text { min }}-P_{i}\right)\right)\right|
\end{array}\right)
\end{aligned}
$$

where $F_{T}$ is total fuel cost of generation in $(\$ / \mathrm{hr})$ including valve point loading, $e_{i}, f_{i}$ are fuel cost coefficients of the $i$ th generating unit reflecting valvepoint effects.



Fig. 1: Valve-point effect

\section{Particle Swarm Optimization}

\subsection{Overview of Particle Swarm Optimization}

The PSO method was introduced in 1995 by Kennedy and Eberhart [12]. The method is motivated by social behaviour of organis ms such as fish schooling and bird flocking. PSO provides a population-based search procedure in which individuals called particles change their position with time. In a PSO system, particles fly around in a multi dimensional search space. During flight each particles adjust its position according its own experience and the experience of the neighboring particles, making use of the best position encountered by itself and its neighbors.

In the multidimensional space where the optimal solution is sought, each particle in the swarm is moved toward the optimal point by adding a velocity with its position. The velocity of a particle is influenced by three components, namely, inertial, cognitive, and social. The inertial component simulates the inertial 
behavior of the bird to fly in the previous direction. The cognitive component models the memory of the bird about its previous best position, and the social component models the memory of the bird about the best position among the particles. The particles move around the multi-dimensional search space until they find the optimal solution. The modified velocity of each agent can be calculated using the current velocity and the distance from Pbest and Gbest as given below.

$$
\begin{aligned}
V_{i}^{k+1}= & W \times V_{i}^{k}+C_{1} \times r_{1} \times\left(\text { Pbest }_{i}^{k}-X_{i}^{k}\right)+ \\
& C_{2} \times r_{2} \times\left(\text { Gbest }^{k}-X_{i}^{k}\right)
\end{aligned}
$$

where,

$$
\begin{array}{ll}
V_{i}^{k} & \text { velocity of individual } i \text { at iteration } k \\
X_{i}^{k} & \text { position of individual } i \text { at iteration } k \\
W & \text { inertia weight } \\
C_{1}, C_{2} & \text { acceleration coefficients } \\
\text { Pbest }_{i}^{k} & \text { best position of individual } i \text { at iteration } k \\
\text { Gbest }^{k} & \text { best position of the group until iteration } k \\
r_{1}, r_{2} & \text { random numbers between } 0 \text { and } 1
\end{array}
$$

In this velocity updating process, the acceleration coefficients $C_{1}, C_{2}$ and the inertia weight $W$ are predefined and $r_{1}, r_{2}$ are uniformly generated random numbers in the range of $[0,1]$. In general, the inertia weight $W$ is set according to the following equation [13]:

$$
W=W_{\text {max }}-\left(\frac{\left(W_{\text {max }}-W_{\text {min }}\right.}{\text { Iter }_{\text {max }}}\right) \times \text { Iter }
$$

where,

$$
\begin{array}{ll}
W_{\max }, W_{\min } & \text { initial and final weights } \\
\text { Iter }_{\max } & \text { maximum iteration number } \\
\text { Iter } & \text { current iteration number }
\end{array}
$$

The approach using (7) is called "inertia weight approach (IWA)". Using the above equation, a certain velocity, which gradually gets close to Pbest and Gbest can be calculated. The current position (searching point in the solution space), each individual moves from the current position to the next one by the modified velocity in (6) using the following equation:

$$
X_{i}^{k+1}=X_{i}^{k}+V_{i}^{k+1}
$$

where,

$$
\begin{array}{cc}
X_{i}^{k+1} & \text { current position of individual } i \text { at iteration } \\
V_{i}^{k+1} & \text { velocity of individual } i \text { at iteration } k+1
\end{array}
$$$$
k+1
$$

Fig. 2 shows the concept of the searching mechanism of PSO using the modified velocity and position of individual $i$ based on (6) and (8) if the value of $W, C_{1}$, $C_{2}, r_{1}$, and $r_{2}$ are 1 .

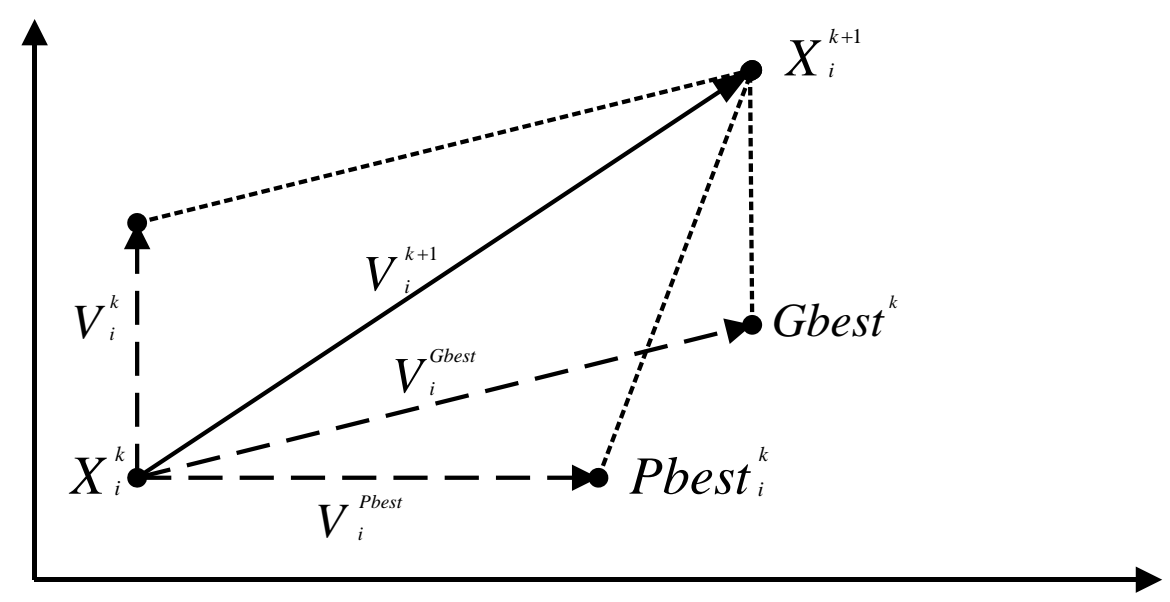

Fig. 2: The search mechanism of the PSO

The process of implementing the PSO is as follows:

Step 1: Create an in itial population of individual with random positions and velocity within the solution space.

Step 2: For each individual, calculate the value of the fitness function.
Step 3: Compare the fitness of each individual with each Pbest. If the current solution is better than its Pbest, then replace its Pbest by the current solution. 
Step 4: Compare the fitness of all individual with Gbest. If the fitness of any individual is better than Gbest, then replace Gbest.

Step 5: Update the velocity and position of all individual according to (6) and (8).

Step 6: Repeat steps 2-5 until a criterion is met.

\subsection{Modified Particle Swarm Optimization}

Coelho and Krohling [18] proposed the use of truncated Gaussian and Cauchy probability distribution to generate random numbers for the velocity updating equation of PSO. In this paper, new approaches to PSO are proposed which are based on Gaussian probability distribution $(G d)$ and Cauchy probability distribution $(C d)$. In this new approach, random numbers are generated using Gaussian probability function and/or Cauchy probability function in the interval $[0,1]$.

The Gaussian distribution $(G d)$, also called normal distribution is an important family of continuous probability distributions. Each member of the family may be defined by two parameters, location and scale: the mean and the variance respectively. A standard normal distribution has zero mean and variance of one. Hence importance of the Gaussian distribution is due in part to the central limit theorem. Since a standard Gaussian distribution has zero mean and variance of value one, it helps in a faster convergence for local search.

Here the Cauchy distribution $C d$, is used to generate random numbers in the interval $[0,1]$, in the social part and Gaussian distribution $G d$, is used to generate random numbers in the interval $[0,1]$ in the cognitive part. The modified velocity equation (6) is given by

$$
\begin{aligned}
& V_{i}^{k+1}=K \cdot\left(\begin{array}{l}
W \cdot V_{i}^{k}+C_{1} G_{d}()\left(\text { Pbest }_{i}^{k}-X_{i}^{k}\right) \\
+C_{2} C_{d}()\left(G b e s t^{k}-X_{i}^{k}\right)
\end{array}\right) \\
& K=\frac{2}{\left|2-\varphi-\sqrt{\varphi^{2}-4 \varphi}\right|}
\end{aligned}
$$

where $\varphi=C_{1}+C_{2}, \varphi>4$.

The convergence characteristic of the system can be controlled by $\varphi$. In the constriction factor approach (CFA), $\varphi$ must be greater than 4.0 to guarantee stability. However, as $\varphi$ increases, the constriction factor $K$ decreases and diversification is reduced, yielding slower response. Typically, when the constriction factor is used, $\varphi$ is set to 4.1 (i.e. $C_{1}, C_{2}=$ 2.05) and the constant multiplier $K$ is thus 0.729.

\section{Results and Discussion}

To verify the feasibility of the proposed method, three different power systems were tested: (1) 6-unit system with valve-point effects and trans mission losses, (2) 13-unit system with valve-point effects and transmission losses are neglected and (3) 40-unit system with valve-point effects and transmission losses are neglected.

\section{Test Case 1: 6-unit system}

The system consists of six thermal generating units with valve point effects. The total load demand on the system is $1263 \mathrm{MW}$. The parameters of all thermal units are presented in Table 1 [14], followed by coefficient matrix $B_{\mathrm{ij}}$ losses.

The obtained results for the 6-unit system using the MPSO method are given in Table 2 and the results are compared with other methods reported in literature, including GA, PSO, PSO-LRS, NPSO, and NPSO-LRS [19]. It can be observed that MPSO can get total generation cost of $15,441(\$ / \mathrm{hr})$ and power losses of $12.216(\mathrm{MW})$, which is the best solution among all the methods. Note that the outputs of the generators are all within the generator's permissible output limit.

Test Case 2: 13-unit system

This system consists of 13 generating units and the input data of 13-generator system are given in Table 3 [10]. In order to validate the proposed MPSO method, it is tested with 13-unit system having non-convex solution spaces. The 13-unit system consists of thirteen generators with valve-point loading effects and have a total load demands of $1800 \mathrm{MW}$ and $2520 \mathrm{MW}$, respectively.

The best fuel cost result obtained from proposed MPSO and other optimization algorithms are compared in Table 4 and Table 5 for load demands of $1800 \mathrm{MW}$ and $2520 \mathrm{MW}$, respectively. In Table 4, generation outputs and corresponding cost obtained by the proposed MPSO are compared with those of DEC-SQP, NN-EPSO, and EP-EPSO [20]. The proposed MPSO provide better solution (total generation cost of $17517.0118 \$ / \mathrm{hr}$ ) than other methods while satisfying the system constraints. In Table 5, generation outputs and corresponding cost obtained by the proposed MPSO are compared with those of GA-SA, EP-SQP, and PSOSQP [20].

The proposed MPSO provide better solution (total generation cost of $24019.8924 \$ / \mathrm{hr}$ ) than other methods while satisfying the system constraints. We have also observed that the solutions by MPSO always are satisfied with the equality and inequality constraints by using the proposed constraint-handling approach.

Test Case 3: 40-unit system

This system consisting of 40 generating units and the input data for 40-generator system is given in Table 6 [10]. The total demand is set to $10,500 \mathrm{MW}$. 
The obtained results for the 40-unit system using the MPSO method are given in Table 7 and the results are compared with other methods reported in literature, including PSO, PPSO, and APPSO [21]. It can be observed that MPSO can get total generation cost of $121,649.20 \$ / \mathrm{hr}$, which is the best solution among all the methods. These results show that the proposed methods are feasible and indeed capable of acquiring better solution.

The optimal dispatches of the generators are listed in Table 7. Also note that all generators' outputs are within its permissible limits.

Table 1: Generating units capacity and coefficients (6-units)

\begin{tabular}{|c|c|c|c|c|c|c|c|}
\hline Unit & $P_{i}^{\min }(\mathbf{M W})$ & $P_{i}^{\max }(\mathbf{M W})$ & $\mathbf{a}$ & $\mathbf{b}$ & $\mathbf{c}$ & $\mathbf{e}$ & $\mathbf{f}$ \\
\hline 1 & 100 & 500 & 0.0070 & 7.0 & 240 & 300 & 0.035 \\
\hline 2 & 50 & 200 & 0.0095 & 10.0 & 200 & 200 & 0.042 \\
\hline 3 & 80 & 300 & 0.0090 & 8.5 & 220 & 200 & 0.042 \\
\hline 4 & 50 & 150 & 0.0090 & 11.0 & 200 & 150 & 0.063 \\
\hline 5 & 50 & 200 & 0.0080 & 10.5 & 220 & 150 & 0.063 \\
\hline 6 & 50 & 120 & 0.0075 & 12.0 & 190 & 150 & 0.063 \\
\hline
\end{tabular}

$$
\begin{aligned}
& B_{i j}=\left[\begin{array}{ccccccc}
0.0017 & 0.0012 & 0.0007 & -0.0001 & -0.0005 & -0.0002 \\
0.0012 & 0.0014 & 0.0009 & 0.0001 & -0.0006 & -0.0001 \\
0.0007 & 0.0009 & 0.0031 & 0.0000 & -0.0010 & -0.0006 \\
-0.0001 & 0.0001 & 0.0000 & 0.0024 & -0.0006 & -0.0008 \\
-0.0005 & -0.0006 & -0.0010 & -0.0006 & 0.0129 & -0.0002 \\
-0.0002 & -0.0001 & -0.0006 & -0.0008 & -0.0002 & 0.0150
\end{array}\right]
\end{aligned}
$$

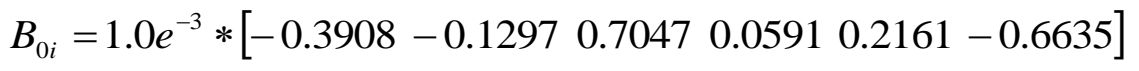

$$
\begin{aligned}
& B_{00}=0.0056
\end{aligned}
$$

Table 2: Comparison of the best results of each methods $\left(\mathrm{P}_{\mathrm{D}}=1263 \mathrm{MW}\right)$

\begin{tabular}{|c|c|c|c|c|c|c|}
\hline Unit Output & GA & PSO & PSO-LRS & NPSO & NPSO-LRS & MPSO \\
\hline P1 (MW) & 474.8066 & 447.4970 & 447.4440 & 447.4734 & 446.9600 & 447.1874 \\
\hline P2 (MW) & 178.6363 & 173.3221 & 173.3430 & 173.1012 & 173.3944 & 173.5060 \\
\hline P3 (MW) & 262.2089 & 263.0594 & 263.3646 & 262.6804 & 262.3436 & 260.9553 \\
\hline P4 (MW) & 134.2826 & 139.0594 & 139.1279 & 139.4156 & 139.5120 & 144.0583 \\
\hline P5 (MW) & 151.9039 & 165.4761 & 165.5076 & 165.3002 & 164.7089 & 163.2156 \\
\hline P6 (MW) & 74.1812 & 87.1280 & 87.1698 & 87.9761 & 89.0162 & 86.2934 \\
\hline Total power output (MW) & 1276.03 & 1276.01 & 1275.95 & 1275.95 & 1275.94 & 1275.216 \\
\hline Total generation cost $(\$ / h r)$ & 15,459 & 15,450 & 15,450 & 15,450 & 15,450 & $\mathbf{1 5 , 4 4 1}$ \\
\hline Power losses $(\mathrm{MW})$ & 13.0217 & 12.9584 & 12.9571 & 12.9470 & 12.9361 & $\mathbf{1 2 . 2 1 6 0}$ \\
\hline
\end{tabular}


Table 3: Generating units capacity and coefficients (13-units)

\begin{tabular}{|c|c|c|c|c|c|c|c|}
\hline Unit & $\mathbf{P}_{\min }(\mathbf{M W})$ & $\mathbf{P}_{\max }(\mathbf{M W})$ & $\mathbf{a}$ & $\mathbf{b}$ & $\mathbf{c}$ & $\mathbf{e}$ & $\mathbf{f}$ \\
\hline 1 & 0 & 680 & 0.00028 & 8.10 & 550 & 300 & 0.035 \\
\hline 2 & 0 & 360 & 0.00056 & 8.10 & 309 & 200 & 0.042 \\
\hline 3 & 0 & 360 & 0.00056 & 8.10 & 307 & 200 & 0.042 \\
\hline 4 & 60 & 180 & 0.00324 & 7.74 & 240 & 150 & 0.063 \\
\hline 5 & 60 & 180 & 0.00324 & 7.74 & 240 & 150 & 0.063 \\
\hline 6 & 60 & 180 & 0.00324 & 7.74 & 240 & 150 & 0.063 \\
\hline 7 & 60 & 180 & 0.00324 & 7.74 & 240 & 150 & 0.063 \\
\hline 8 & 60 & 180 & 0.00324 & 7.74 & 240 & 150 & 0.063 \\
\hline 9 & 60 & 180 & 0.00324 & 7.74 & 240 & 150 & 0.063 \\
\hline 10 & 40 & 120 & 0.00284 & 8.60 & 126 & 100 & 0.084 \\
\hline 11 & 40 & 120 & 0.00284 & 8.60 & 126 & 100 & 0.084 \\
\hline 12 & 55 & 120 & 0.00284 & 8.60 & 126 & 100 & 0.084 \\
\hline 13 & 55 & 120 & 0.00284 & 8.60 & 126 & 100 & 0.084 \\
\hline
\end{tabular}

Table 4: Comparison of the best results of each methods $\left(\mathrm{P}_{\mathrm{D}}=1800 \mathrm{MW}\right)$

\begin{tabular}{|c|c|c|c|c|}
\hline Unit power output & DEC-SQP [20] & NN-EPSO [20] & EP-EPSO [20] & MPSO \\
\hline P1 (MW) & 526.1823 & 490.0000 & 505.4731 & 425.0980 \\
\hline P2 (MW) & 252.1857 & 189.0000 & 254.1686 & 182.5087 \\
\hline P3 (MW) & 257.9200 & 214.0000 & 253.8022 & 133.5717 \\
\hline P4 (MW) & 78.2586 & 160.0000 & 99.8350 & 162.4450 \\
\hline P5 (MW) & 84.4892 & 90.0000 & 99.3296 & 153.9582 \\
\hline P6 (MW) & 89.6198 & 120.0000 & 99.3035 & 113.9438 \\
\hline P7 (MW) & 88.0880 & 103.0000 & 99.7772 & 133.8305 \\
\hline P8 (MW) & 101.1571 & 88.0000 & 99.0317 & 104.7926 \\
\hline P9 (MW) & 132.0983 & 104.0000 & 99.2788 & 85.6033 \\
\hline P10 (MW) & 40.0007 & 13.0000 & 40.0000 & 66.7367 \\
\hline P11 (MW) & 40.0000 & 58.0000 & 40.0000 & 60.8971 \\
\hline P12 (MW) & 55.0000 & 66.0000 & 55.0000 & 77.3235 \\
\hline P13 (MW) & 55.0000 & 55.0000 & 55.0000 & 99.2915 \\
\hline Total power output (MW) & 1800 & 1800 & 1800 & 1800 \\
\hline Total generation cost (\$/h) & 17938.9521 & 18442.5931 & 17932.4766 & $\mathbf{1 7 5 1 7 . 0 1 1 8}$ \\
\hline
\end{tabular}

Table 5: Comparison of the best results of each methods $\left(P_{D}=2520 \mathrm{MW}\right)$

\begin{tabular}{|c|c|c|c|c|}
\hline Unit power output & GA-SA [20] & EP-SQP [20] & PSO-SQP [20] & MPSO \\
\hline P2 (MW) & 299.22 & 299.0524 & 299.0524 & 322.2105 \\
\hline P3 (MW) & 299.17 & 299.0474 & 298.9681 & 319.4067 \\
\hline P4 (MW) & 159.12 & 159.6399 & 159.4680 & 170.7089 \\
\hline P5 (MW) & 159.95 & 159.6560 & 159.1429 & 136.4957 \\
\hline P7 (MW) & 157.26 & 159.6749 & 159.5371 & 128.8908 \\
\hline P8 (MW) & 159.93 & 159.7265 & 158.8522 & 131.4204 \\
\hline P9 (MW) & 159.86 & 159.6653 & 159.7845 & 158.3310 \\
\hline $\mathrm{P} 10(\mathrm{MW})$ & 110.78 & 114.0334 & 110.9618 & 117.6114 \\
\hline Total power output (MW) & 2520 & 2520 & 2520 & 2520 \\
\hline Total generation cost $(\$ / \mathrm{h})$ & 24275.71 & 24266.44 & 24261.05 & 24019.8924 \\
\hline
\end{tabular}


Table 6: Generating units capacity and coefficients (40-units)

\begin{tabular}{|c|c|c|c|c|c|c|c|}
\hline Unit & $\mathbf{P}_{\min }(\mathbf{M W})$ & $P_{\max }(M W)$ & $\mathbf{a}$ & $\mathbf{b}$ & c & e & $\mathbf{f}$ \\
\hline 1 & 36 & 114 & 0.00690 & 6.73 & 94.705 & 100 & 0.084 \\
\hline 2 & 36 & 114 & 0.00690 & 6.73 & 94.705 & 100 & 0.084 \\
\hline 3 & 60 & 120 & 0.02028 & 7.07 & 309.54 & 100 & 0.084 \\
\hline 4 & 80 & 190 & 0.00942 & 8.18 & 369.03 & 150 & 0.063 \\
\hline 5 & 47 & 97 & 0.01140 & 5.35 & 148.89 & 120 & 0.077 \\
\hline 6 & 68 & 140 & 0.01142 & 8.05 & 222.33 & 100 & 0.084 \\
\hline 7 & 110 & 300 & 0.00357 & 8.03 & 287.71 & 200 & 0.042 \\
\hline 8 & 135 & 300 & 0.00492 & 6.99 & 391.98 & 200 & 0.042 \\
\hline 9 & 135 & 300 & 0.00573 & 6.60 & 455.76 & 200 & 0.042 \\
\hline 10 & 130 & 300 & 0.00605 & 12.9 & 722.82 & 200 & 0.042 \\
\hline 11 & 94 & 375 & 0.00515 & 12.9 & 635.20 & 200 & 0.042 \\
\hline 12 & 94 & 375 & 0.00569 & 12.8 & 654.69 & 200 & 0.042 \\
\hline 13 & 125 & 500 & 0.00421 & 12.5 & 913.40 & 300 & 0.035 \\
\hline 14 & 125 & 500 & 0.00752 & 8.84 & 1760.4 & 300 & 0.035 \\
\hline 15 & 125 & 500 & 0.00708 & 9.15 & 1728.3 & 300 & 0.035 \\
\hline 16 & 125 & 500 & 0.00708 & 9.15 & 1728.3 & 300 & 0.035 \\
\hline 17 & 220 & 500 & 0.00313 & 7.97 & 647.85 & 300 & 0.035 \\
\hline 18 & 220 & 500 & 0.00313 & 7.95 & 649.69 & 300 & 0.035 \\
\hline 19 & 242 & 550 & 0.00313 & 7.97 & 647.83 & 300 & 0.035 \\
\hline 20 & 242 & 550 & 0.00313 & 7.97 & 647.81 & 300 & 0.035 \\
\hline 21 & 254 & 550 & 0.00298 & 6.63 & 785.96 & 300 & 0.035 \\
\hline 22 & 254 & 550 & 0.00298 & 6.63 & 785.96 & 300 & 0.035 \\
\hline 23 & 254 & 550 & 0.00284 & 6.66 & 794.53 & 300 & 0.035 \\
\hline 24 & 254 & 550 & 0.00284 & 6.66 & 794.53 & 300 & 0.035 \\
\hline 25 & 254 & 550 & 0.00277 & 7.10 & 801.32 & 300 & 0.035 \\
\hline 26 & 254 & 550 & 0.00277 & 7.10 & 801.32 & 300 & 0.035 \\
\hline 27 & 10 & 150 & 0.52124 & 3.33 & 1055.1 & 120 & 0.077 \\
\hline 28 & 10 & 150 & 0.52124 & 3.33 & 1055.1 & 120 & 0.077 \\
\hline 29 & 10 & 150 & 0.52124 & 3.33 & 1055.1 & 120 & 0.077 \\
\hline 30 & 47 & 97 & 0.01140 & 5.35 & 148.89 & 120 & 0.077 \\
\hline 31 & 60 & 190 & 0.00160 & 6.43 & 222.92 & 150 & 0.063 \\
\hline 32 & 60 & 190 & 0.00160 & 6.43 & 222.92 & 150 & 0.063 \\
\hline 33 & 60 & 190 & 0.00160 & 6.43 & 222.92 & 150 & 0.063 \\
\hline 34 & 90 & 200 & 0.00010 & 8.95 & 107.87 & 200 & 0.042 \\
\hline 35 & 90 & 200 & 0.00010 & 8.62 & 116.58 & 200 & 0.042 \\
\hline 36 & 90 & 200 & 0.00010 & 8.62 & 116.58 & 200 & 0.042 \\
\hline 37 & 25 & 110 & 0.01610 & 5.88 & 307.45 & 80 & 0.098 \\
\hline 38 & 25 & 110 & 0.01610 & 5.88 & 307.45 & 80 & 0.098 \\
\hline 39 & 25 & 110 & 0.01610 & 5.88 & 307.45 & 80 & 0.098 \\
\hline 40 & 242 & 550 & 0.00313 & 7.97 & 647.83 & 300 & 0.035 \\
\hline
\end{tabular}


Table 7: Comparison of the best results of each methods $\left(\mathrm{P}_{\mathrm{D}}=10,500 \mathrm{MW}\right)$

\begin{tabular}{|c|c|c|c|c|}
\hline Unit power output & PSO [21] & PPSO [21] & APPSO [21] & MPSO \\
\hline P1 (MW) & 113.116 & 111.601 & 112.579 & 113.9971 \\
\hline P2 (MW) & 113.010 & 111.781 & 111.553 & 112.6517 \\
\hline P3 (MW) & 119.702 & 118.613 & 98.751 & 119.4255 \\
\hline P4 (MW) & 81.647 & 179.819 & 180.384 & 189.0000 \\
\hline P5 (MW) & 95.062 & 92.443 & 94.389 & 96.8711 \\
\hline P6 (MW) & 139.209 & 139.846 & 139.943 & 139.2798 \\
\hline P7 (MW) & 299.127 & 296.703 & 298.937 & 223.5924 \\
\hline P8 (MW) & 287.491 & 284.566 & 285.827 & 284.5803 \\
\hline P9 (MW) & 292.316 & 285.164 & 298.381 & 216.4333 \\
\hline P10 (MW) & 279.273 & 203.859 & 130.212 & 239.3357 \\
\hline P11 (MW) & 169.766 & 94.283 & 94.385 & 314.8734 \\
\hline P12 (MW) & 94.344 & 94.090 & 169.583 & 305.0565 \\
\hline P13 (MW) & 214.871 & 304.830 & 214.617 & 365.5429 \\
\hline P14 (MW) & 304.790 & 304.173 & 304.886 & 493.3729 \\
\hline P15 (MW) & 304.563 & 304.467 & 304.547 & 280.4326 \\
\hline P16 (MW) & 304.302 & 304.177 & 304.584 & 432.0717 \\
\hline P17 (MW) & 489.173 & 489.544 & 498.452 & 435.2428 \\
\hline P18 (MW) & 491.336 & 489.773 & 497.472 & 417.6958 \\
\hline P19 (MW) & 510.880 & 511.280 & 512.816 & 532.1877 \\
\hline P20 (MW) & 511.474 & 510.904 & 548.992 & 409.2053 \\
\hline P21 (MW) & 524.814 & 524.092 & 524.652 & 534.0629 \\
\hline P22 (MW) & 524.775 & 523.121 & 523.399 & 457.0962 \\
\hline P23 (MW) & 525.563 & 523.242 & 548.895 & 441.3634 \\
\hline P24(MW) & 522.712 & 524.260 & 525.871 & 397.3617 \\
\hline P25 (MW) & 503.211 & 523.283 & 523.814 & 446.4181 \\
\hline P26 (MW) & 524.199 & 523.074 & 523.565 & 442.1164 \\
\hline P27 (MW) & 10.082 & 10.800 & 10.575 & 74.8622 \\
\hline P28 (MW) & 10.663 & 10.742 & 11.177 & 27.5430 \\
\hline P29 (MW) & 10.418 & 10.799 & 11.210 & 76.8314 \\
\hline P30 (MW) & 94.244 & 94.475 & 96.178 & 97.0000 \\
\hline P31(MW) & 189.377 & 189.245 & 189.999 & 118.3775 \\
\hline P32 (MW) & 189.796 & 189.995 & 189.924 & 188.7517 \\
\hline P33 (MW) & 189.813 & 188.081 & 189.714 & 190.0000 \\
\hline P34 (MW) & 199.797 & 198.475 & 199.284 & 120.7029 \\
\hline P35 (MW) & 199.284 & 197.528 & 199.599 & 170.2403 \\
\hline P36 (MW) & 198.165 & 196.971 & 199.751 & 198.9897 \\
\hline P37 (MW) & 109.291 & 109.161 & 109.973 & 110.0000 \\
\hline P38 (MW) & 109.087 & 109.900 & 109.506 & 109.3405 \\
\hline P39 (MW) & 109.909 & 109.855 & 109.363 & 109.9243 \\
\hline P40 (MW) & 512.348 & 510.984 & 511.261 & 468.1694 \\
\hline Total generation cost $(\$ / \mathrm{h})$ & $122,323.97$ & $121,788.22$ & $122,044.63$ & $121,649.20$ \\
\hline
\end{tabular}




\section{Conclusion}

This paper presents a new approach for solving ELD problems with valve-point effect using a modified particle swarm optimization (MPSO) technique. The MPSO technique has provided the global solution in the 6-unit, 13-unit, and 40-unit test system and the better solution than the previous studies reported in literature. The application of Gaussian and Cauchy probability distributions in MPSO is a powerful strategy to improve the global searching capability and escape from local minima. Also, the equality and inequality constraints treatment methods have always provided the solutions satisfying the constraints. Although the proposed MPSO algorithm had been successfully applied to ELD with valve-point loading effect, the practical ELD problems should consider multiple fuels as well as prohibited operating zones. This remains a challenge for future work.

\section{References}

[1] A. J Wood and B. F. Wollenberg, Power Generation, Operation, and Control, $2^{\text {nd }}$ ed., John Wiley and Sons, New York, 1996.

[2] Z. X. Liang and J. D. Glover. A Zoom Feature for a Dynamic Programming Solution to Economic Dispatch Including Transmission Losses, IEEE Trans. on Power Systems, 1992, 7(2): 544-550.

[3] P. H. Chen and H. C. Chang. Large-Scale Economic Dispatch by Genetic Algorithm, IEEE Trans. Power Systems, 1995, 10(4): 1919-1926.

[4] C. L. Chiang. Improved Genetic Algorithm for Power Economic Dispatch of Units with ValvePoint Effects and Multiple Fuels, IEEE Trans. Power Systems, 2005, 20(4): 1690-1699.

[5] W. M. Lin, F. S. Cheng and M. T. Tsay. An Improved Tabu Search for Economic Dispatch with Multiple Minima, IEEE Trans. Power Systems, 2002, 17(1): 108-112.

[6] K. P. Wong and C. C. Fung. Simulated Annealing Based Economic Dispatch Algorithm, Proc. Inst. Elect. Eng. C, 1993, 140(6): 509-515.

[7] J. H. Park, Y. S. Kim, I. K. Eom and K. Y. Lee. Economic Load Dispatch for Piecewise Quadratic Cost Function using Hopfield Neural Network, IEEE Trans. Power Systems, 1993, 8(3): 10301038.

[8] K. Y. Lee, A. Sode-Yome and J. H. Park. Adaptive Hopfield Neural Network for Economic Load Dispatch, IEEE Trans. Power Systems, 1998, 13(2): 519-526.

[9] T. Jayabarathi and G. Sadasivam. Evolutionary Programming-Based Economic Dispatch for Units with Multiple Fuel Options, European Trans. Elect. Power, 2000, 10(3): 167-170.

[10] N. Sinha, R. Chakrabarti and P. K. Chattopadhyay. Evolutionary Programming Techniques for Economic Load Dispatch, IEEE Trans. Evolutionary Computation, 2003, 7(1): 83-94.

[11] H. T. Yang, P. C. Yang and C. L. Huang. Evolutionary Programming Based Economic Dispatch for Units with Non-Smooth Fuel Cost Functions, IEEE Trans. Power Systems, 1996, 11(1): 112-118.

[12] J. Kennedy and R. Eberhart. Particle Swarm Optimization, in Proc. IEEE Int. Conf. Neural Networks (ICNN'95), Perth, Australia, 1995, IV: 1942-1948.

[13] Y. Shi and R. Eberhart. A Modified Particle Swarm Optimizer, Proceedings of IEEE International Conference on Evolutionary Computation, Anchorage, Alaska, 1998, 69-73.

[14] Z. L. Gaing. Particle Swarm Optimization to Solving the Economic Dispatch Considering the Generator Constraints, IEEE Trans. Power Systems, 2003, 18(3): 1187-1195.

[15] J. B. Park, K. S. Lee, J. R. Shin and K. Y. Lee. A Particle Swarm Optimization for Economic Dispatch with Non-Smooth Cost Functions, IEEE Trans. Power Systems, 2005, 20(1): 34-42.

[16] J. B. Park, Y. W. Jeong, J. R. Shin, K. Y. Lee and J. H. Kim. A Hybrid Particle Swarm Optimization Employing Crossover Operation for Economic Dispatch Problems with Valve-Point Effects, Engineering Intelligent Systems for Electrical Engineering and Communications, 2007, 15(2): 29-34.

[17] Shi Yao Lim, Mohammad Montakhab and Hassan Nouri. Economic Dispatch of Power System Using Particle Swarm Optimization with Constriction Factor, International Journal of Innovations in Energy Systems and Power, 2009, 4(2): 29-34.

[18] L. S. Coelho and C.S. Lee. Solving Economic Load Dispatch Problems in Power Systems Using Chaotic and Gaussian Particle Swarm Optimization Approaches, Electric Power and Energy Systems, 2008, 30: 297-307.

[19] A. I. Selvakumar and K. Thanushkodi. A New Particle Swarm Optimization Solution to Nonconvex Economic Dispatch Problems, IEEE Trans. Power Systems, 2007, 22(1): 42-51.

[20] S. Muthu Vijaya Pandian and K. Thanushkodi. An Evolutionary Programming Based Efficient Particle Swarm Optimization for Economic Dispatch Problem with Valve-Point Loading, European Journal of Scientific Research, 2011, 52(3): 385-397. 
[21] C.H. Chen and S. N. Yeh. Particle Swarm Optimization for Economic Power Dispatch with Valve-Point Effects, IEEE PES Transmission and Distribution Conference and Exposition Latin America, Venezuela, 2006.

\section{Authors' Profiles}

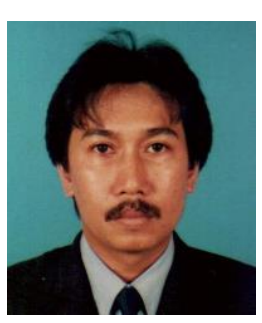

Hardiansyah was born on February 27, 1967 in Mempawah, Indonesia. He received the B.S. degree in Electrical Engineering from the University of Tanjungpura in 1992 and the M.S. degree in Electrical Engineering from Bandung Institute of Technology (ITB), Indonesia in 1996. Dr. Eng, degree from Nagaoka University of Technology in 2004.

Since 1992, He has been with Department of Electrical Engineering University of Tanjungpura, Pontianak, Indonesia, where he is a lecturer. His current research interests include power system dynamics and controls, robust control, and power systemanalysis.

How to cite this paper: Hardiansyah,"A Modified Particle Swarm Optimization Technique for Economic Load Dispatch with Valve-Point Effect", International Journal of Intelligent Systems and Applications(IJISA), vol.5, no.7, pp.32-41, 2013. DOI: $10.5815 /$ ijisa.2013.07.05 